\title{
Development of Materials and Processes for Non-top Coat Immersion Lithography
}

\author{
Katsutoshi Kobayashi, Kotaro Sho, Hirokazu Kato, Kazunori Iida, Yoko Iwakaji, \\ Tomoya Ori, Daizo Muto, Tsukasa Azuma and Shinichi Ito
}

\author{
Process \& Manufacturing Engineering Center, \\ Toshiba Corporation Semiconductor Company, \\ 8, Shinsugita-cho, Isogo-ku, Yokohama 235-8522, Japan \\ katsutoshi2.kobayashi@toshiba.co.jp
}

\begin{abstract}
Non-top coat resists are expected to be compatible with next generation high-speed scanners. Since they contain hydrophobic additives which are eccentrically located near the film surface so that they can form more hydrophobic film surface, they should have more suitable performance for high-speed scanning as well as better cost performance because of their processes without any immersion top coat steps. However, the influence of the addition of hydrophobic additives to the resists on their lithographic and defect performance need to be investigated, in order to apply them as mass production processes. In this work, the influence of hydrophobic additives on the scanning performance and on the imaging performance is investigated. Moreover, the influnece of global resist pattern density on a wafer on defect performance is also investigated. In addition, the defect performance is demonstrated to be sucessfully improved by improving the development and rinse processes.
\end{abstract}

Keywords: non-top coat resist, immersion lithography, top coat

1. Introduction

The miniaturization of the semiconductor device is effective to improve the performance, the function, and the reliability. The semiconductor device pattern shrinkage has been accomplished by shortening wavelength of the exposure light source and introducing the higher NA (numerical aperture) lens. Recently, development of immersion lithography technology has realized much higher NA lens such as 1.3-1.35 NA. In order to improve mass productivity, high-throughput scanners with higher scan speed such as $600-700 \mathrm{~mm} / \mathrm{sec}$ are under development. As resist processes compatible with such high-speed scanners, non-top coat resist processes are required ${ }^{(1)}$. Since the non-top coat resists contain hydrophobic additives which are eccentrically located near the film surface so that they can form more hydrophobic film surface ${ }^{(2)(4)}$, they should have more suitable performance for high-speed scanning as well as better cost performance because of their processes without any immmersion top coat steps. However, the influence of the addition of hydrophobic additives to the resists on their lithographic and defect performance should be investigated, in order to apply them as mass production processes. In this work, the influences of imaging performance, scanning performance and defect performance of the non-top coat resists were evaluated. 


\section{Experimental conditions}

Table 1 shows compositions of top coat and non-top coat resist materials evaluated in this work. Resist A is a conventional ArF resist using immersion top coat. Resists B, C, D and E have the same base polymer X that resist $\mathrm{A}$ and the same hydrophobic additives type $\alpha$ and the different additive concentrations. The concentrations of the hydrophobic additives are normalized as resist $\mathrm{B}$ is 1.0. Resist $\mathrm{F}$ has different base polymer Y.

Table 1. Compositions of top coat and non-top coat resist materials evaluated in this work.

\begin{tabular}{|l|l|l|l|l|l|l|}
\hline Resist & A & B & C & D & E & F \\
\hline $\begin{array}{l}\text { Base } \\
\text { Polymer }\end{array}$ & X & X & X & X & X & Y \\
\hline $\begin{array}{l}\text { Additive } \\
\text { Type }\end{array}$ & w/o & $\alpha$ & $\alpha$ & $\alpha$ & $\alpha$ & $\alpha$ \\
\hline $\begin{array}{l}\text { Additive } \\
\text { Concentration (a.u.) }\end{array}$ & w/o & 1.0 & 1.25 & 1.5 & 2.1 & 1.3 \\
\hline
\end{tabular}
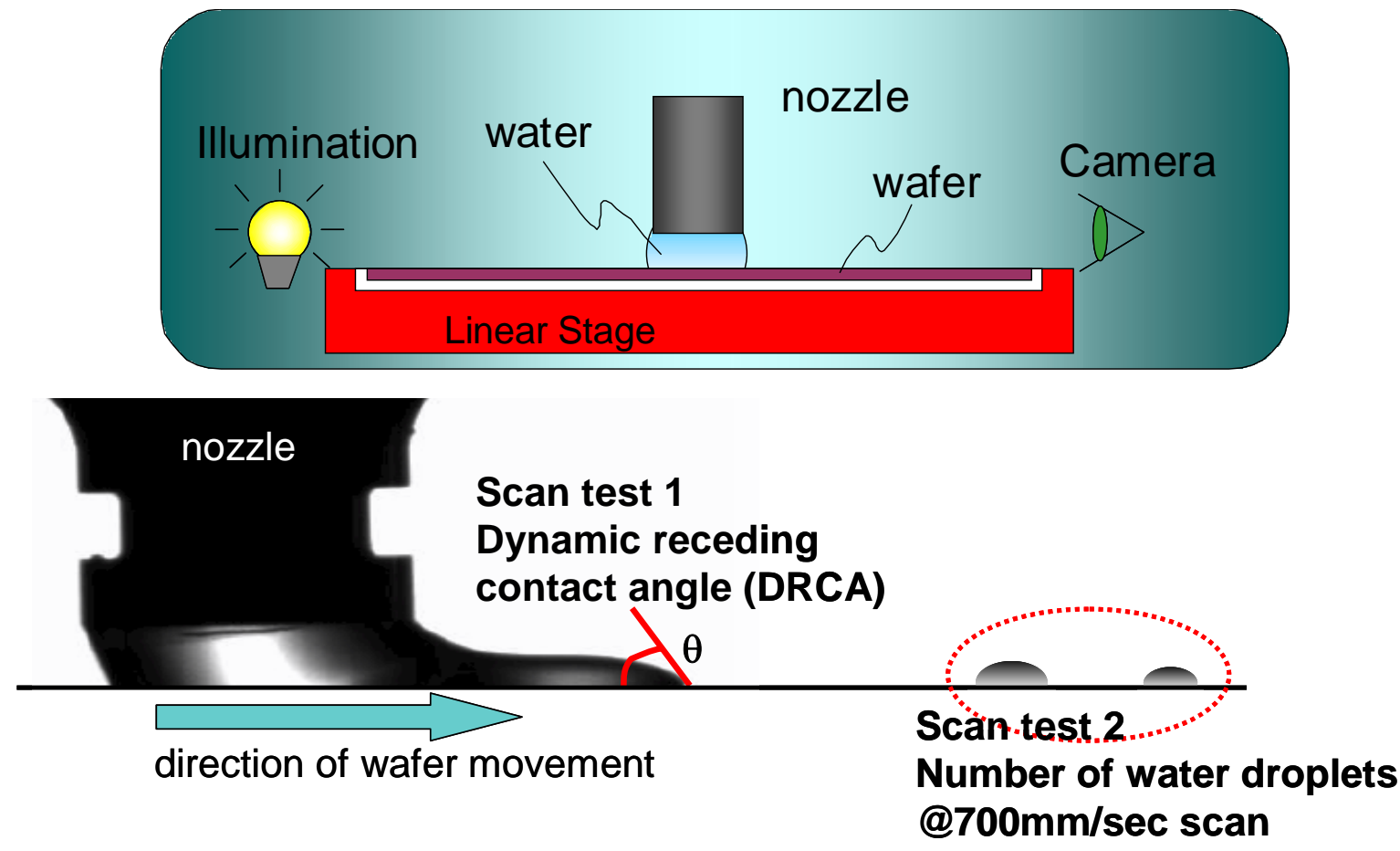

Fig 1. Schematic diagram of scanning test tool.

\subsection{Scanning performance}

The non-top coat resists were spin-coated on bare-Si wafers. Scanning test was performed using a scanning test tool with an immersion nozzle. Schematic diagram of the scanning test tool is shown in Fig. 1. By scanning the nozzle on non-top coat resists, dynamic receding contact angle (DRCA) was measured, and the number of water droplets on the surface of non-top coat resists was counted.

\subsection{Imaging performance}

Under-layer materials were applied on bare-Si wafers, and then the non-top coat resists were coated over the under-layer materials. Nikon NSR-S610C and ASML TWINSCAN XT1900i were used as immersion scanners. 43nm L/S patterns were exposed using an illumination condition of 1.3 NA. CD (critical dimension), LWR (line width roughness) and pattern collapse margin were measured by a CD-SEM, Hitachi High-Technology CG4000 and a cross-sectional SEM, 
Hitachi High-Technology S-5500.

\subsection{Defect performance}

Defects were inspected by an inspection tool, KLA-Tencor KLA2815, and then reviewed defects using a review SEM tool, Applied Materials SEMVision G4.

\section{Results \& Discussion}

3.1. Results of scanning performance

Scanning test results using resist $\mathrm{D}$ and resist $\mathrm{F}$ were shown in Table 2 and Fig. 2. DRCA increased as increasing concentration of hydrophobic additives. In Fig. 2 , broken line indicates the upper limit of defect count. In the water droplets test at $700 \mathrm{~mm} / \mathrm{sec}$ scanning, the number of water droplets was less than spec $(<5)$ in case of resist $\mathrm{D}\left(\mathrm{DRCA}=70^{\circ}\right.$ ) and in case of resist $\mathrm{F}$ (DRCA $=62.9^{\circ}$ ). Resist $\mathrm{D}$ and resist $\mathrm{F}$ showed better scanning performance. It was concluded that better scanning performance required DRCA more than $60^{\circ}$.

Table 2. Scanning test results

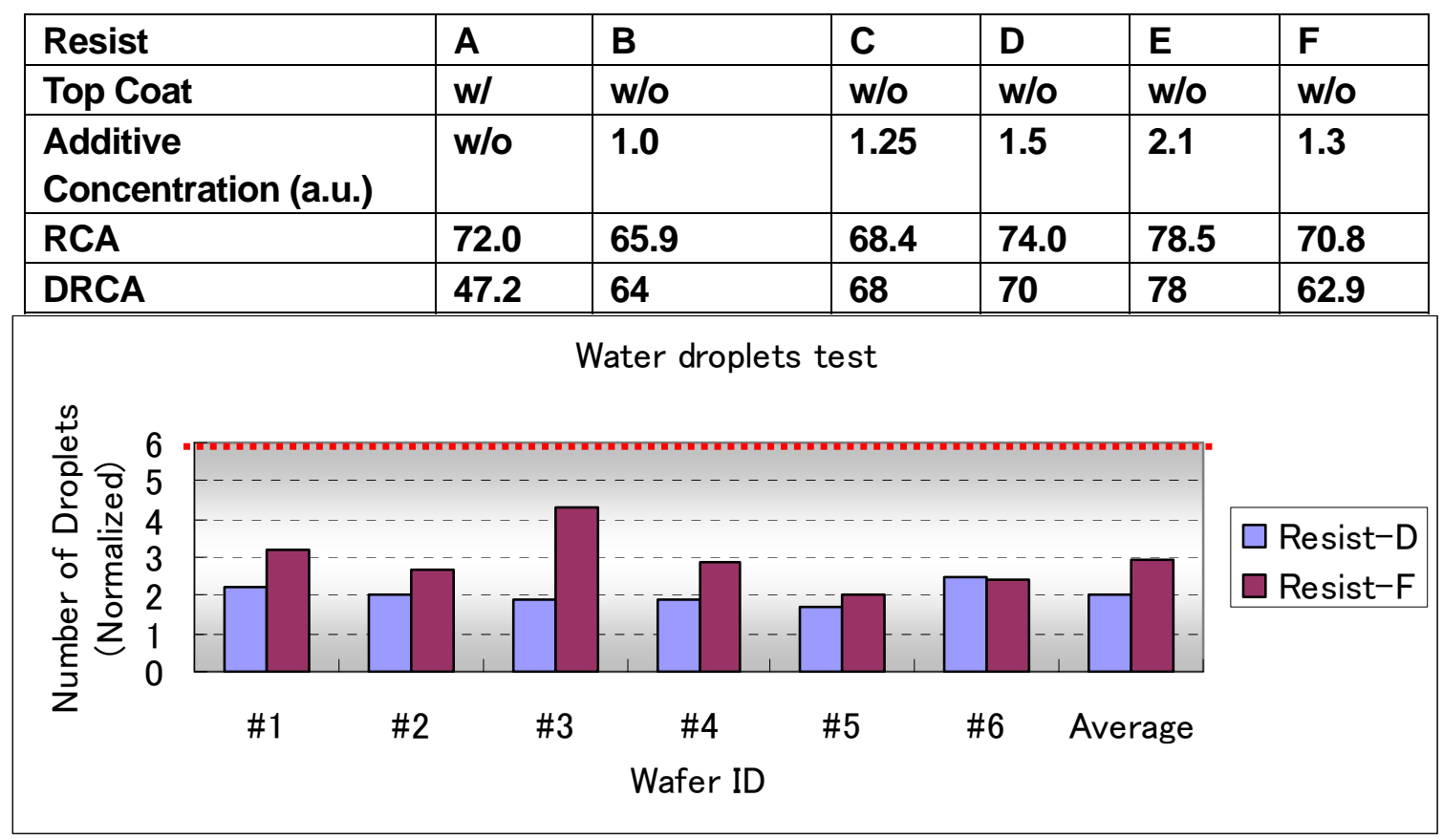

Fig 2. Results of water droplets test using resist $\mathrm{D}$ and resist $\mathrm{F}$.

\subsection{Results of imaging performance}

Results of imaging performance were shown in Table 3. Pattern collapse margins were deteriorated with increasing concentration of the hydrophobic additives. Remaining resist thickness after development tended to increase with increasing concentration of the hydrophobic additives. Photo-sensitivity decreased with increasing concentration of the hydrophobic additives. Max E.L. (exposure latitude) and DoF (depth of focus) were almost same in all resists 
Table 3. Results of imaging performance

\begin{tabular}{|l|l|l|l|l|l|}
\hline Resist & A & B & C & D & E \\
\hline Top Coat & wl & w/o & w/o & w/o & w/o \\
\hline LWR (nm) & 4.24 & 4.10 & 4.25 & 4.42 & 4.23 \\
\hline $\begin{array}{l}\text { Pattern Collapse } \\
\text { Margin (nm) }\end{array}$ & 34.3 & 38.1 & 38.7 & 39.3 & 40.4 \\
\hline $\begin{array}{l}\text { Remaining Resist } \\
\text { Thickness (nm) }\end{array}$ & 92.6 & 91.3 & 90.0 & 92.6 & 95.2 \\
\hline Max E.L. (\%) & 13.7 & 11.4 & 13.2 & 12.5 & 13.8 \\
\hline DoF (nm) & $>360$ & $>330$ & $>360$ & $>330$ & $>330$ \\
\hline Eop (mJcm-^2) & 13.49 & 14.71 & 14.75 & 14.73 & 14.75 \\
\hline
\end{tabular}

\subsection{Results of defect performance}

\subsubsection{Comparison with a top coat process}

When defect performance of the non-top coat process using resist $F$ was compared with that of top coat process, defect density (D.D.) of the non-top coat process tended to be higher than that of the top coat process. In the top coat process, D.D. was $0.23 \mathrm{~cm}^{-2}$. On the other hand, in the non-top coat process, it was $6.60 \mathrm{~cm}^{-2}$. From review SEM images of the defects, it was found that the major defects were blob defects, as shown in Fig. 3. It is considered that the blob defects were hard to be completely removed in rinse process because the hydrophobic properties of the non-top coat resists were too high.
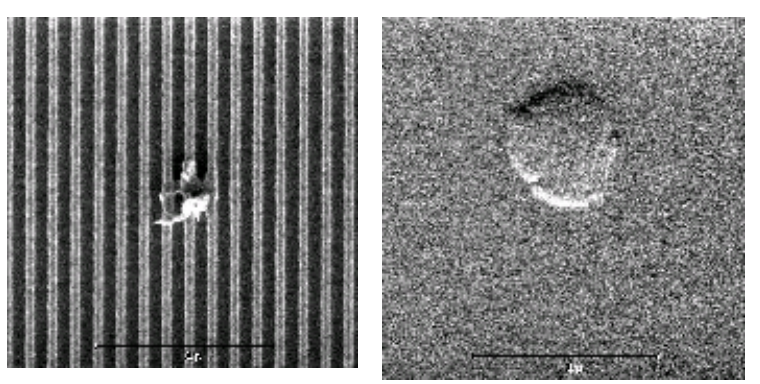

Fig. 3. SEM images of blob defects

\subsubsection{Influence of global resist pattern density}

The influence of global resist pattern density on a wafer on defect performance was estimated. Defects were increased with increasing the global resist pattern density. In 75\% global resist pattern density, D.D. was $10.6 \mathrm{~cm}^{-2}$, and in $87.5 \%$ global resist pattern density, it was $33.9 \mathrm{~cm}^{-2}$, as shown in Fig. 4. It is considered that the wettability of developer and rinse water was poorer with increasing global resist pattern density.

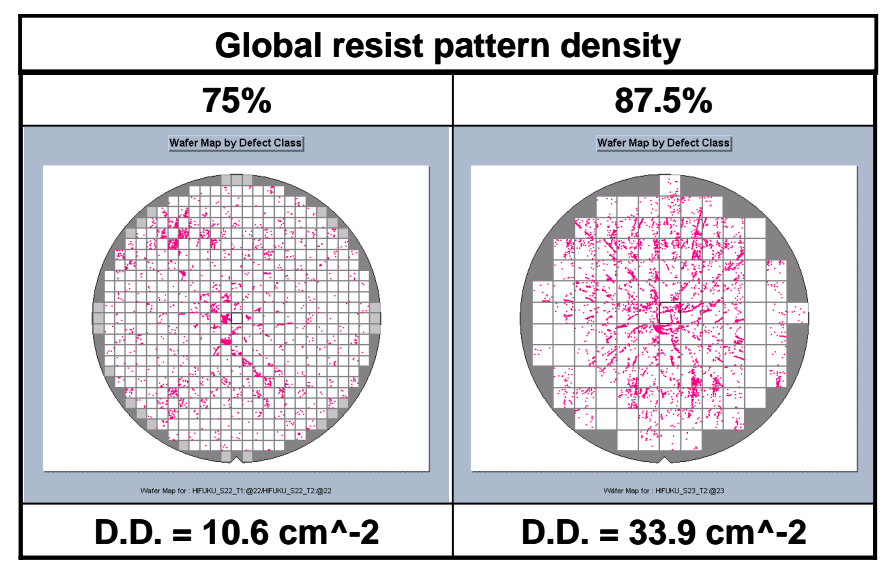

Fig. 4. Defect map images

\subsubsection{Improvement of defect performance}

In order to reduce the defect density, the development and rinse processes were optimized. The processes were changed so that the surface of the non-top coat resists could be covered with the developer and rinse water, and so that the rinse process could produce no defects. The defect performance could be improved from D.D. $=58.8 \mathrm{~cm}^{-2}$ to D.D. $=1.12 \mathrm{~cm}^{-2}$. In addition, the D.D. could be improved less than $0.80 \mathrm{~cm}^{-2}$ by applying a post soak process. The post soak process, which is a treatment process after immersion exposure, could reduce not only the blob defects but also water mark defects as shown in Fig. 5. 


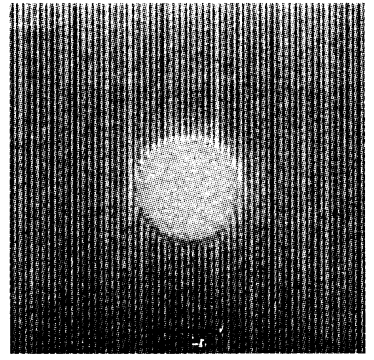

Fig. 5. SEM images of water mark defects

\section{Conclusion}

From the evaluation of scanning performance, DRCA increased with increasing concentration of the hydrophobic additives. And, resist $\mathrm{D}$ and resist $\mathrm{F}$ showed better scanning performance at $700 \mathrm{~mm} / \mathrm{sec}$ scanning. Moreover, from the evaluation of imaging performance, pattern collapse margins were deteriorated with increasing concentration of the hydrophobic additives. Finally, from the evaluation of defect performance, the defects were increased with increasing the global resist pattern density. In addition, the defect density could be reduced by improving the development and rinse processes, and also by applying the post soak process.

\section{Acknowledgments}

We wish to express our gratitude to FUJIFILM Co. which supplied the non-top coat resist materials, and Nikon Co. which helped in the scanning test. We would like to thank Mr. M. Hatano, Toshiba Co., for his useful advices in the scanning performance test.

\section{References}

1. N. Matsumura, N. Sugie, K. Goto, K. Fujiwara and Y. Yamaguchi, H. Tanizaki, K. Nakano and T. Fujiwara, S. Wakamizu, H. Takeguchi, H. Arima, H. Kyoda, K. Yoshihara and J. Kitano, Proc. SPIE, 2008, 6923, 69230D (2008).

2. K. Ohmori, T. Ando, T. Takayama, K. Ishizuka, M. Yoshida, Y. Utsumi, K. Endo, T. Iwai. Proc. SPIE, 6153, 61531X (2006).

3. M. Irie, K. Endo, T. Iwai. J. Photopol. Sci. Technol.,, 19, 565 (2006)

4. D. P. Sanders, L. K. Sundberg, R. Sooriyakumaran, P. J. Brock, R. A. Dipietro, H. D. Truong, D. C. Miller, M . C. Lawson, R. D. Allen, Proc. SPIE, 6519, 651904 (2007) 